\title{
LES MÉTAPHORES DE L'IMMUNOLOGIE: GUERRE ET PAIX
}

\author{
METAPHORS OF IMMUNOLOGY: \\ WAR AND PEACE
}

Ilana Löwy*

LÖWY, I.: 'Metaphors of immunology: war and peace'. História, Ciências, Sauide-Manguinhos, III (1):7-23, Mar.-Jun. 1996.

Immunology has always relied on metaphorical language. In its early beginnings, it varied between bellicose images and images that stressed the interaction of immunity mechan isms with the organism's physiological functions overall. In the late nineteenth century, white blood globules were not only compared to 'border police' assigned to rebuffing intruders - an army formed to combat the invaders but were also described as a physiological mechanism for eliminating aged, dead cells, at times exterminators of foreign bodies.

Antibodies were described as very powerful, deadly weapons but also as an integral part of mechanisms that allowed cells to assimilate food. This duality of images holds true today. The article analyzes the emergence and development of these images, relating them to the redefinition of immunology as a science of the self and non-self and dissecting them in light of recent events, stuch as the Aids epidemic.

KEYWORDS: immunologie, metaphors, regulation, infection, history of science.

* Ilana lowy est directeur de recherche à l'Unité 158 de I Institut de la Santéet de la Recherche Médicale (INSERM) $\mathrm{U}-158$ INSERM

Hôpital Necker-Enfants Malades

149, rue de Sèves, 74743

Paris-Cedex 15

França
La popularisation du savoir immunologique passe par des images de guerre: certains globules blancs sont présents comme des 'sentinelles' guettant l'arrivée de 'l'ennemi' (un micro-organisme pathogène) et alertant d'autres cellules capables de détruire l'envahisseur, le site d'inflammation est décrit comme un champ de bataille rangée; il existe même l'équivalent de services de contre-espionnage: des 'lymphocytes tueurs' qui s'attaquent à 'l'ennemi intérieur' - les cellules cancéreuses. Ces images bien que très efficaces - n'ont nullement été fabriquées dans l'optique d'expliquer à un public non-spécialiste des phénomènes immunologiques complexes. Les métaphores guerrières sont nées avec l'immunologie moderne et sont inséparables de l'imaginaire immunologique. Elles n'ont cependant pas l'exclusivité des représentations en immunologie: dès les débuts de cettescience, des métaphores de combat ont coexisté avec une perception de 
l'immunité comme mécanisme physiologique de base et avec des images qui mettent l'immunité en relation avec d'autres mécanismes physiologiques: digestion, sécrétion, perception. Les deux modes de représentations de l'immunité ont été souvent si étroitement entremêlés qu'il n'est pas rare de les voir se côtoyer dans une même page d'un livre d'immunologie. Cependant, il ne faut pas en déduire l'existence de rapports symétriques entre les deux. Tandis que les métaphores de guerre, simples et puissantes, ont toujours gardé une place importante dans l'imaginaire des immunologistes, la vision de l'immunité comme mécanisme physiologique a connu une longue période d'éclipse, faute de cadre théorique adéquat.

L'association de l'immunologie á des métaphores guerrières, et notamment á l'image du corps qui combat une armée d'envahisseurs étrangers et menaçants, trouve ses origines dans les débuts de l'immunologie comme 'science pasteurienne'. Le terme ancien d'immunité' — donc exemption - des maladies infectieuses est aussi, il est vrai, emprunté au langage militaire; pourtant, il n'évoque pas l'image active du combat avec un ennemi, mais plutôt celle, passive, de l'existence de différences et de hiérarchies entre les individus. Le langage guerrier a pris définitivement racine en immunologie á un moment très précis: 1890. Cette année-là, Pasteur a invité le zoologiste russe Ilia Metchnikoff, auteur d'une théorie sur le rôle des cellules phagocytaires dans la défense antimicrobienne, à venir s'installer à l'Institut Pasteur récemment inauguré (1888), afin d'y ouvrir un service consacré à l'étude des mécanismes de résistance aux maladies infectieuses. C'est vers 1884 que Metchnikoff a développé sa théorie sur le rôle des cellules phagocytaires dans la défense antimicrobienne de l'organisme. Cette théorie s'est inspirée des réflexions de Metchnikoff sur le rôle des cellules mésodermiques mobiles, dotées de la capacité d'engloutir des particules dans l'embryogenèse. Si l'on ajoute foi au récit rétroactif de Metchnikoff sur les origines de sa découverte, elle a été aussi directement inspirée par sa perception de l'analogie entre la propension des cellules amiboïdes mobiles chez la larve de l'étoile de mer à s'agglutiner autour d'un corps étranger et l'accumulation de cellules blanches autour d'une écharde dans le doigt. La théorie metchnikovienne de l'inflammation affirmait que "les activités digestives des cellules mobiles du mésoderme servent à protéger l'organisme des bactéries", et que l'inflammation doit être perçue avant tout comme une lutte entre les cellules phagocytaires et les microbes.

L'utilisation par Metchnikoff d'un langage de lutte n'est pas surprenante chez ce darwinien convaincu, bien que nonorthodoxe. Un tel langage pouvait pourtant paraître primitif et 
${ }^{1}$ Cité par L. J. Rather (1970). Virchow a protesté contre la vision "clatée" et "pré-scientifique" de la physiopathologie propagée en Allemagne pendant la première moitié clu $\mathrm{XIX}^{\mathrm{e}}$ siècle par les savants appartenant au courant romantique, et qui a employé des termes tels que "la guerre contre la maladie" ou "forces défensives de l'organisme". (Alfred I. Tauber et Leon Chernyak, 1991). simpliste á certains savants. Virchow (qui, plus tard, s'est d'ailleurs rallié aux théories de Metchnikoff) a ainsi rejeté en 1847 une théorie qui voulait faire des globules blancs du sang les responsables de l'élimination des substances nuisibles: "Nous ne considérons plus les corpuscules du pus comme des gendarmes qui sont commandés par l'État policier pour raccompagner à la frontière un étranger dépourvu de passeport." ${ }^{1}$ Semblable argumentation a été avancée contre Metchnikoff par ses critiques (principalement allemands) partisans des théories chimiques de l'inflammation. Le langage guerrier de Metchnikoff a, en revanche, beaucoup plus à Pasteur, et pour cause: il y a une proximité très grande entre les langages métaphoriques de ces deux savants. De plus, quand Pasteur a proposé sa théorie de l'origine bactérienne des maladies infectieuses, il s'est vu accusé de retour à des théories dépassées. En effet, les théories qui ont attribué aux organismes vivants la capacité d'induire les maladies ont été perçues comme 'primitives' au regard des théories 'scientifiques' de l'origine chimique des maladies.

Pasteur a trouvé dans les théories de Metchnikoff une solution satisfaisante au problème de l'immunité antibactérienne. Avant d'adopter une vision active de l'immunité, Pasteur a conçu l'immunité en termes passifs: d'abord (1880) comme le résultat de l'épuisement, par les bactéries, des substances nutritives essentielles: "J'ai envisagé l'organisme comme un milieu de culture qui, par une première atteinte du mal, perdrait, sous l'influence de la culture du parasite, des principes que la vie n'y ramènerait pas ou n'y ramènerait qu'après un certain temps", puis (1886) comme le résultat de la sécrétion, par le microbe, de substances qui lui sont toxiques. Les deux théories étaient peu satisfaisantes, et n'ont pas pu être vérifiées expérimentalement. La théorie phagocytaire, qui opte pour une réaction active de l'organisme aux micro-organismes pathogènes, s'harmonisait à merveille avec l'imagerie pasteurienne classique de l'invasion du corps par des troupes étrangères. En outre, elle se prêtait à d'impressionnantes démonstrations visuelles: on pouvait littéralement voir sous le microscope comment les phagocytes avalent, puis détruisent les bactéries virulentes.

Vers 1890 , une autre théorie active de l'immunité fut proposée - la théorie humorale. Cette théorie (dont le porte-parole le plus important était le savant allemand Paul Ehrlich) opérait, elle aussi, avec des images guerrières fondées sur le principe de la lutte de l'organisme contre l'envahisseur. La principale différence entre les deux imageries concernait la nature des armes employées par l'organisme dans la guerre contre les bactéries: tandis que les partisans (principalement français) de la théorie cellulaire de l'immunité choisissaient l'image d'une lutte au corps à corps entre 
bactéries et phagocytes, les partisans (principalement allemands) d'une théorie humorale de l'immunité préféraient celle d'une attaque d'artillerie par missiles dirigés - les anticorps (les "balles magiques" d'Ehrlich). Les deux écoles ont cependant utilisé un vocabulaire fort semblable. En 1901, Metchnikoff explique qu'en rencontrant une bactérie pathogène, "les phagocytes entrent en lutte contre ces micro-organismes et débarrassent l'organisme de ces envahisseurs", tandis qu'en 1907, Ehrlich affirme que

"dans tous les cas, il y a une lutte entre l'adaptabilité du parasite et de l'hôte. ... Cette lutte est, évidemment, influencée par la sécrétion, par chaque antagoniste, de substances solubles qui sont hostiles à la vitalité et la réceptivité de chaque organisme. Côté bactérie, ces substances sont des toxines; côté organisme, des anti-substances (des anticorps, I. L.) De plus, il y a un rôle déterminé pour des actions défensives et protectrices. .... Vous voyez donc qu'il s'agit d'une guerre menée dans des sphères différentes, mais dans laquelle il y a une réaction á chaque action."

La prédominance des images guerrières dans la description des maladies infectieuses n'était pas forcément du goût de tout le monde. Le médecin et philosophe de la médecine polonais Wladyslaw Bieganski, partisan d'une perception fonctionnelle de la maladie, a vigoureusement critiqué, dans son livre Problèmes généraux de la théorie des sciences médicales de 1897, le langage guerrier en vogue:

"Si la maladie est une perturbation des fonctions induite par l'action d'un stimulus pathologique, il est totalement dépourvu de sens de parler à ce sujet d'une lutte, de même qu'il est dépourvu de sens d'exprimer de cette manière notre compréhension d'un phénomène physiologique. Personne n'affirmera que la digestion est une lutte de l'organisme contre la nourriture; personne ne devrait affirmer que l'inflammation est une telle lutte. ... La maladie n'est donc pas une lutte, mais une réaction de l'organisme aux stimuli, et la différence entre lutte et réaction est énorme. Le concept de lutte est celui d'une action indépendante et délibérée, tandis que la réaction est seulement soumise aux lois de la chimie et de la physique. Les réactions chimiques sont bien connues. Il serait pourtant d'une grande licentia poetica que de parler de luttes en chimie. ... Nous n'aurions pas eu d'objection à une utilisation purement métaphorique du mot 'lutte' dans la compréhension des maladies. Ceci n'est pourtant pas le cas. La maladie est perçue comme une véritable lutte, et même comme une bataille rangée entre une armée de phagocytes et des bactéries. Une telle perception est décidément erronée." 
Les idées de Bieganski étaient moins éloignées de celles des fondateurs de l'immunologie qu'il pourrait y paraitre. Metchnikoffet Ehrlich ont eu, il est vrai, recours à des images de guerre entre l'organisme et les microbes, mais en même temps, ils ont perçu les réactions immunitaires comme une fonction particulière des mécanismes physiologiques les plus importants. Ainsi pour Metchnikoff, la destruction des bactéries par les phagocytes était l'une, parmi quantité d'autres, des fonctions physiologiques de ces cellules. Les phagocytes étaient responsables de l'élimination des cellules mortes, vieilles ou inutiles. Ils avaient donc un rôle clé dans le fonctionnement de l'organisme. Ehrlich, pour sa part, a envisagé les anticorps comme des "chaînes latérales" du protoplasme, i.e. des molécules dont le rôle principal est de réagir aux substances nutritives et d'assurer leur assimilation par la cellule. C'est seulement accessoirement que ces "chaînes latérales" aident l'organisme à se débarrasser des substances nuisibles présentes en grande quantité, telles les toxines bactériennes.

Le classique de l'immunologie, Méthodes d'immunodiagnostic et d'immunothérapie de Citron, publié en 1910, résume la perception physiologique des réactions immunitaires (tout en reprenant, lui aussi, des métaphores guerrières). Dans sa préface, Citron affirme:

"Nous savons maintenant que les réactions d'immunité humorale, comme celles d'immunité cellulaire, ne sont pas limitées au champ des maladies infectieuses mais sont, dans une beaucoup plus grande mesure, l'expression de phénomènes physiologiques, soit normaux, soit pathologiques. Le concept astucieux de chaînes latérales, développé par Ehrlich, nous a permis de comprendre que les manifestations physiologiques de l'assimilation, qui fonctionnent dans la nutrition et la consommation de l'énergie, correspondent aux événements qui, dans des circonstances pathologiques, conduisent à la formation de produits antiinfectieux. Metchnikoff a montré, dans un travail non moins remarquable, que le même groupe de cellules originaires du mésenchyme et que l'organisme mobilise contre un ennemi bactérien, remplit une variété de tâches physiologiques et patho-physiologiques dans le royaume animal. Elles coopèrent à la métamorphose de la structure du corps des animaux inférieurs en faisant disparaître des organes entiers. Elles prennent part à l'involution de l'utérus aprés l'accouchement, font disparaître des cellules nerveuses pendant l'atrophie sénile des centres nerveux, et, sous la forme de chromophages, elles blanchissent les cheveux comme signe du progrès de l'âge. Il n'est donc pas possible de tracer avec précision la ligne qui divise les événements normaux et patbologiques: il s'agit plutôt d'une chaîne de phénoménes avec des transitions multiples." 
Une telle vision physiologique de l'immunité peut être retrouvée dans les écrits d'autres pionniers de l'immunologie. Ainsi l'élève et collaborateur de Metchnikoff, Jules Bordet, explique que "la science de l'immunité évolue, elle rencontre, en s'étendant, le domaine plus vaste de la physiologie générale. Le champ qu'elle explore en tous sens, la défense de l'organisme contre les microbes, est pour elle un point de départ. Peut-être, un jour, semblera-t-il lointain."

Une vision fort originale de la signification physiologique de l'immunité fut proposée vers 1911 par Charles Richet. Ce savant a découvert l'anaphylaxie - une réaction immune violente, parfois mortelle, après un deuxième contact avec une substance sensibilisatrice - et a tenté d'expliquer la signification physiologique de ce phénomène, en apparence si nuisible pour l'individu. Il en a proposé deux explications. La première prolongeait la vision habituelle de l'immunité comme mécanisme de défense. L'anaphylaxie, explique Richet, est un mécanisme de protection du patrimoine génétique d'une espèce: "Si des substances hétérogènes pouvaient impunément pénétrer dans l'organisme et modifier ses caractères chimiques fondamentaux, pénétrant dans le protoplasma pour en altérer la nature, alors c'en serait fait de la constitution somatique de chaque espèce animale, fruit d'une lente et ancestrale acquisition." Mais, ajoute Richet, en plus de leur rôle dans la défense contre des micro-organismes pathogènes et dans la sauvegarde du patrimoine génétique de l'espèce, les mécanismes d'immunité et d'anaphylaxie déterminent l'individualité biologique:

"L'anaphylaxie, comme l'immunisation, créent la différence humorale entre les individus. ... Au cours de la vie, chaque organisme, qui est unique, accumulera des immunisations ou des sensibilités anaphylactiques qui sont lui propres. ... Chacun de nous, par la composition chimique, avant tout par le sang mais probablement aussi par la structure de chaque cellule, est luimême et personne d'autre. En d'autres mots, il a une personnalité humorale. Nous savons tous ce qu'est la personnalité psychique. ... Maintenant, à la lumière de l'immunité et de l'anaphylaxie, nous pouvons concevoir une autre personnalité, juxtaposée à la personnalité psychique - une personnalité humorale qui nous rend différent des autres êtres humains par la constitution chimique de nos humeurs. ... Chaque être vivant, même s'il présente la plus grande ressemblance avec les autres individus de son espèce, a ses caractéristiques propres, et est lui-même et personne d'autre. Ceci signifie que l'étude de la physiologie des espéces ne suffit plus. Une étude d'une autre physiologie doit être entreprise, une étude fort difficile et à peine ébauchée, celle de la physiologie de l'individu."Richet, Citron et Bordet (qui a 
pourtant vécu jusqu'en 1961) appartiennent à la première génération d'immunologistes, fortement influencée par une vision physiologique de l'immunité. Cette vision a fini par disparaître avec l'abandon des théories de Metchnikoff et d'Ehrlich désormais perçues comme démodées - sur les liens entre l'immunité et les fonctions physiologiques fondamentales, telles la sécrétion ou la digestion. Ces théories ne furent pourtant pas remplacées par des théories nouvelles. Les immunologistes, découragés par le vide théorique de leur discipline et par la difficulté des mécanismes cellulaires complexes, se sont tournés vers des systèmes expérimentaux simples et reproductibles, telle la réaction d'antigènes et d'anticorps en tube à essai. L'entredeux-guerres vit le développement rapide d'approches étudiant la production d'anticorps contre des antigènes bien définis: sérologie médicale d'une part, immunochimie d'autre part. Dans le même temps, les études des réactions physiologiques complexes (allergie, anaphylaxie, phénomènes auto-immunes) sont reléguées à une position marginale. L'imaginaire immunologique est resté fidèle aux images de bataille entre l'organisme et les bactéries (par "balles magiques" interposées), mais lors de la période 'chimique'de l'immunologie, cette bataille s'est déroulée sur une grande place vide.

Le tournant immunochimique a été perçu par la majorité des immunologistes comme un signe de maturation de leur discipline. Certains, pourtant, ont critiqué le divorce grandissant entre l'immunologie et la physiologie.

"Le résultat de la faillite de l'ancienne théorie de l'immunité et de l'absence d'une théorie alternative, l'absence de liens entre la communauté des sérologistes et celle des non-spécialistes et le rôle dominant de la technique dans cette discipline, explique en 1937 l'immunologiste et épistémologue Ludwik Fleck, les termes fondamentaux de l'immunologie, tels 'complément', 'amboceptor', 'composante A-B', 'réactif sérologique' etc., ont acquis une qualité d'apostasie, i.e. la réification des états, des réactions et même des manipulations de laboratoire. Cette réification est maintenant devenue une caractéristique constante de la sérologie."

Les promoteurs des approches chimiques, poursuit Fleck, ont tendance à s'enfermer dans des représentations simplistes:

"Parmi les concepts classiques de l'immunologie, beaucoup ont été développés pendant la période où, sous l'influence d'importants, succès chimiques en physiologie, des tentatives erronées ont été faites pour expliquer l'ensemble, ou presque, de la biologie en termes d'effets produits par des substanceschimiquement bien définies. Toxines, amboceptors et compléments sont traités comme 
des entités chimiques distinctes par des ennemis tels que antitoxines et anticompléments."

La solution proposée par Fleck consista à renouveler les bases théoriques de l'immunologie, en substituant à l'approche fondée sur la chimie une approche fondée sur les acquis de la biophysique et de la physique moderne:

"Le schéma primitif, fondé sur les substances activatrices et inhibitrices, est progressivement dévalorisé par les théories physico-chimiques et colloïdales provenant d'autres domaines. Nous parlons maintenant d'états ou de structures plutôt que de substances, afin d'exprimer la possibilité qu'un état complexe chimico-physico-morphologique est responsable de la modification des réactions, et non des substances bien définies chimiquement ou de leur mélange."

L'idée qu'un renouveau des théories biologiques viendra de la physique moderne (et, inversement, que l'étude des phénomènes biologiques contribuera à la découverte de nouvelles lois physiques) fut propagée, dans les années 1935, par des physiciens de grande renommée: Erwin Schrödinger et Max Delbruck. Si cette idée n'a mené in fine ni à la découverte, en physique, de nouvelles lois, ni à la régression, en biologie, de l'importance de l'étude des réactions chimiques, elle a, en revanche, précipité les événements qui ont conduit à la naissance de la biologie moléculaire. Cependant, contrairement aux prédictions de Fleck, le renouveau de l'immunologie n'est pas venu du côté de son alliance avec la physique. Il est venu, d'une part, du retour des immunologistes aux grandes questions biologiques (les mécanismes de mémoire immunologique et la reconnaissance du soi) et d'autre part, de l'intérêt renouvelé des immunologistes pour certains problèmes de la pathologie. Un chercheur a joué un rôle clé dans cette réorientation de l'immunologie dans les années 1940 et 50 - l'Australien Macfarlane Burnet.

Burnet aimait se présenter comme l'un des derniers représentants d'une espèce en voie de disparition - celle des 'médecins naturalistes'. En même temps, il a suivi de près l'évolution des nouvelles approches dans l'étude du vivant, comme les théories sur la synthèse des protéines ou sur les liens entre gènes et enzymes. La dualité des ses intérêts l'a mené à une tentative fort originale: la traduction de la perception physiologique des phénomènes de l'immunité dans le nouveau langage de l'immunochimie. L'avènement de l'immunochimie n'a jamais fait disparaître l'intérêt pour la physiologie des réactions immunitaires. Cet intérêt correspondait aux préoccupations 
2 Anne Marie Moulin et Arthur M. Silverstein (1989); Arthur M. Silverstein (1989). Plus tard, les études 'confuses' des phénomènes pathologiques compliqués furent opposées, dans l'histoire officielle de l'immunologie, aux attitudes 'scientifiques'des immunochimistes qui ont étudié cles réactions reproductibles et aisément quantifiables (Meclawar, 1977).

3 E.g., lat théorie a utocatalytique de synthèse des protéines proposée par Bergmann et Niemann (1937). des médecins qui ont étudié les phénomènes pathologiques liés à l'immunité - le rejet de greffe, l'auto-immunité, les déficits immunitaires. Dans les années 1920 et 30, de telles préoccupations furent cependant laissées aux marges de l'immunologie. ${ }^{2}$

Burnet s'est emparé des questions clés de l'immunologie en les reformulant dans le langage de la recherche biologique moderne. Dans son livre publié en 1941, La synthèse des anticorps, il déplore que l'immunologie est souvent considérée comme une sous-division de la bactériologie et qu'elle est avant tout perçue comme une activité orientée vers des applications pratiques. Une telle vision de l'immunologie, affirme Burnet, est erronée. Cette discipline pointe plusieurs questions fondamentales de la biologie moderne: la nature des mécanismes qui régissent la synthèse des protéines, l'habilité des systèmes biologiques de se modifier en vertu de leur expérience passée, et la capacité de l'organisme de distinguer - et de réagir contre des éléments que lui sont étrangers. Une théorie de l'immunité devrait lier toutes ces questions et les exprimer dans le langage biochimique et génétique. Ainsi il faudra identifier des éléments vraisemblablement des cellules - qui conservent la 'mémoire immunologique', des mécanismes biochimiques qui l'activent et permettent une synthèse rapide des anticorps (donc, des protéines hautement spécifiques) par ces cellules, ainsi que la base chimique de la réactivité sélective aux molécules reconnues comme 'étrangéres' à l'organisme. L'idée que la 'mémoire immunologique' réside dans des cellules n'était pas neuve. Émile Duclaux avait déjà expliqué, en 1896, que les substances chimiques étaient trop instables pour expliquer la persistance de l'état d'immunité; cet état doit donc étre sauvegardé par la composante fixe des tissus, les cellules. L'originalité de la démarche de Burnet reposait sur le rapprochement entre l'approche physiologique de la production des anticorps et les théories récentes sur la synthèse des protéines. Une fois ce rapprochement accompli, les concepts et les pratiques des immunologistes, jusque-là principalement cantonnés au domaine étroit de la sérologie, devenaient accessibles à d'autres spécialistes: biochimistes, biophysiciens, généticiens - et dignes d'intérêt à leurs yeux. ${ }^{3}$

Dans la deuxième édition de son livre (1949), Burnet a précisé son propos, en intégrant les résultats des investigations sur le rejet de greffe menêes dans les annêes 1940 par le savant anglais Peter Medawar. Medawar démontre qu'une deuxième greffe de peau originaire du même donneur est rejetée plus rapidement que la première. Medawar conclut à un phénomène immunitaire. Cette observation, couplée à des informations sur la 'tolérance' des globules étrangers greffés chez les veaux jumeaux qui onteu une circulation de sang croisé pendant la gestation, a servi de 
4 Ce terme fut proposé par Burnet pour décrire les changements radicaux dans l'immunologie pendant les annés 1950. point de départ à la proposition de Burnet: la tâche première des mécanismes d'immunité est la distinction entre le 'soi' et le 'non-soi' et la réaction à ce dernier. La capacité de distinguer entre le soi et le non-soi est acquise pendant le développement embryonnaire, puis transmise par les lignées de cellules qui ont 'appris' à distinguer la configuration chimique individualisée présente dans chaque cellule. S'inspirant du modèle des groupes érythrocytaires, Burnet envisage l'existence d'un nombre limité de molécules - des "marqueurs de soi" - qui peuvent se combiner à l'infini et définir ainsi l'individualité chimique de l'organisme. Les réactions immunitaires auront donc une composante innée - la combinaison des molécules qui constitue le soi - et une composante acquise - l'apprentissage de la reconnaissance des marqueurs de soi par les cellules impliquées dans les réactions immunitaires.

La vision physiologique de l'immunité fondée sur la distinction entre le soi et le non-soi a été rapidement adoptée par les chercheurs et les médecins. Elle a été confortée par l'expérimentation animale, telle la description de la tolérance acquise chez la souris et d'autre part, elle a été popularisée grâce aux progrès des greffes de rein chez l'homme. Une perception de l'immunitécomme "réaction contre le non-soi" a pu redonner une signification physiologique à une série de phénomènes auparavant difficiles à expliquer, comme la réaction aux antigènes artificiels - un non-soi évident. L'élaboration, dans les années 1955-57, de la théorie clonale de la synthèse des anticorps a consolidé la vision physiologique et cellulaire de l'immunité, introduit la transcription des questions clés de l'immunologie dans des termes compatibles avec les idées nouvelles sur la synthèse des protéines, et facilité l'intégration de l'immunologie dans les sciences biologiques de pointe. Conjuguées, la perception de l'immunité comme mécanisme de discrimination entre le soi et le non-soi et l'explication clonale (donc cellulaire) du fonctionnement de ce mécanisme ont permis le développement de la "nouvelle immunologie" $-{ }^{4}$ une sousspécialité biologique qui perçoit les réactions immunitaires comme des mécanismes physiologiques impliqués dans le fonctionnement normal de l'organisme et dans de nombreux états pathologiques, et qui étudie l'ensemble des mécanismes cellulaires de l'immunité - le "système immunitaire".

Le retour des approches physiologiques à l'étude des phénomènes immunologiques a accru l'intérêt des médecins pour ce domaine. A partir des années 1960, un grand nombre de pathologies humaines ont été décrites comme la conséquence des défaillances des mécanismes de l'immunité. D'autre part, lesmultiples recherches menées dans le cadre de la "nouvelle 
immunologie" ont rapidement révélé la très grande complexité des mécanismes de l'immunité. La nouvelle perception de la complexité du système immunitaire a trouvé son expression la plus cohérente dans la théorie du réseau idiotypique. D'après cette théorie, chaque anticorps peut être en même temps reconnu comme un nouvel antigéne, puisque son site d'interaction avec l'antigéne est une nouvelle structure spatiale, que l'organisme ne connaît pas. La réponse immunitaire résulte donc d'une interaction entre antigèrnes externes et antigènes internes (anticorps ou idiotypes), et sa régulation se fait par des réseaux complexes d'idiotypes, anti-idiotypes, anti-anti-idiotypes etc. La théorie du réseau idiotypique postule aussi que le système immunitaire a évolué au contact du soi plutôt qu'en réaction au monde extérieur. D'après cette théorie, les frontières entre le soi et le non-soi sont sous-déterminées et fluides, et les réactions immunitaires sont ordonnées selon les principes "je est un autre" et "connais-toi toi-même".

Qu'advient-il alors des métaphores guerrières - celles qui stigmatisent une lutte sans merci contre un ennemi extérieur? Avec l'avènement de la "nouvelle immunologie" et de la perception de l'immunité comme systéme physiologique de plus en plus compliqué, furent-elles définitivement abandonnées? Il n'en est rien. La vision de l'immunité comme "lutte de l'organisme contre la maladie" n'a jamais disparu de l'imaginaire des médecins et des malades, elle a continué de coexister avec la nouvelle vision physiologique de cette discipline. Deux exemples récents - le sida et les traitements immunologiques du cancer — nous permettront d'illustrer la cohabitation, dans la médecine actuelle, de la perception de l'immunitécomme mécanisme physiologique avec la vision de l'immunité comme formidable machine de guerre.

L'immunodéficience grave observée vers 1981 dans certaines populations bien déterminées (homosexuels masculins, utilisateurs de drogues injectables, Haitiens, immigrants de l'Afrique centrale) fut initialement perçue comme un syndrome fort compliqué et vraisemblablement multicausal. La découverte qu'il s'agit en fait d'une maladie infectieuse nouvelle a entraîné la prolifération d'images guerrières faisant du sida le résultat de l'attaque d'un virus (le HIV) particulièrement virulent et 'perfide'. Ces images ont induit des descriptions populaires de la maladie par les médias. Elles ont aussi orienté les représentations professionnelles de cette maladie. La pathologie du sida fut analysée comme résultant d'une agression directe des particules virales contre le système immunitaire et le système nerveux, tandis que les efforts pour trouver une thérapeutique ont été centrés - et le sont toujours - sur le développement des traitements capables d'éliminer le 
HIV. Le traitement des infections opportunistes et des tumeurs, tel le sarcome de Kaposi, a été présenté comme incapable, à long terme, de remplacer une véritable thérapie causale - à même de s'attaquer directement à la racine du mal.

La perception du sida comme désordre induit par une cause unique - le HIV - s'est cependant heurtée aux complexités de la pathologie et de l'épidémiologie de cette maladie. L'ensemble des manifestations du sida (les médecins l'ont constaté) est difficilement réductible aux effets connus du HIV sur les cellules humaines. Cette difficulté a conduit certains chercheurs à l'hypothèse que le sida est une maladie auto-immunitaire. Selon cette hypothése, récemment confortée par des résultats expérimentaux (mais qui n'est nullement admise par l'ensemble des professionnels), le HIV porte des antigènes semblables à ceux qu'on trouve à la surface de certaines cellules humaines et de ce fait, est capable de déclencher une cascade de réactions auto-immunitaires aboutissant à l'autodestruction de certaines souspopulations cellulaires. Si elle est confirmée, cette vision des effets liés àl'infection par le HIV présentera une image fort différente de celle de l'attaque d'un ennemi extérieur (le virus) qui s'introduit en cachette dans le sang, puis s'acharne à détruire les cellules qui y circulent. On peut supposer pourtant qu'une perception (auto-immune ou autre) du sida comme résultat d'une interaction compliquée entre facteurs extérieurs et intérieurs n'abolira pas l'image ramenant cette maladie à la conséquence d'une attaque de forces hostiles, et que certaines stratégies de thérapie et de prévention continueront d'être fondées sur des images guerrières.

Si le sida fut successivement perçu comme événement physiologique compliqué, puis comme 'attaque extérieure', enfin récemment replacé sous le signe de la complexité, l'immunothérapie du cancer fut d'emblée conéue en termes d'intervention dans le réseau compliqué des circuits régulateurs. Les molécules utilisées dans la thérapie du cancer - interférons et interleukines - sont décrites comme ayant des activités physiologiques multiples. Cependant, quand ces mêmes molécules - telle l'interleukine-2 - sont utilisées dans la thérapie des cancers, leur image change subitement. Des articles à visée clinique mettent en avant une seule activité de l'interleukine la capacité d'activer les 'cellules tueuses', globules blancs crédités du pouvoir d'éliminer les cellules malignes. Le terme même de cellules tueuses renvoie immédiatement à un vocabulaire guerrier. Ainsi le dr. Steven Rosenberg, pionnier de l'application de l'interleukine-2 à la thérapie du cancer, explique que les cellules tueuses activées par l'interleukine "ressemblentà n'importe quel autre lymphocyte, mais ce sont en vérité des armes nouvelles contre 
5 En France, des photographies (quasi identiques) de 'cellules tueuses' s'attaquant à une cellule tumorale ont servi à illustrer des articles sur les nouvelles immunothérapies du cancer (L'Express, 17.12.1987; Le Nouvel Observateur, 1.2.1989; Le Figaro Magazine, 5.5.1990; Le Figaro Magazine, 5.12.1992).

6 Jacobi et Schiele (1992) ont analysé en détail la rhétorique de la vulgarisation du traitement par interleukine-2 maniée par un magazine français.

7 Jean-François Lyotard (1988) a défini le postmodernisme comme une rupture avec les représentations en vogue qui allèguent l'irreprésentable dans la représentation ellemême. Cette définition permettra à Lyotard de classer Proust et Montaigne comme 'post-modernes'. le cancer, capables de reconnaître et d'attaquer une tumeur particuliére".

Des récits de vulgarisation qui exposent les nouvelles thérapies du cancer rendent tangible le vocabulaire guerrier par le truchement des images de microscope de balayage. Dans ces images, on aperçoit d'abord une cellule cancéreuse (en général très grande et de forme irrégulière) entourée de plusieurs cellules rondes - les 'lymphocytes tueurs'. Sur les photos suivantes, la même cellule 'monstrueuse' est montré déformée et trouée, vaincue par les vaillants 'soldats' appartenant à l'armée de défense du corps. ${ }^{5}$ Ces représentations guerrières ne sont cependant nullement réservées au grand public. Dans des publications destinées aux professionnels, certains avocats de l'immunothérapie du cancer ont eu recours à un langage semblable, bien que plus nuancé. D'autre part, des récits de vulgarisation peuvent eux aussi jouer sur les deux registres: les interleukines qui 'arment' les cellules tueuses sont en même temps présentées comme des 'substances naturelles' qui activent les mécanismes physiologiques et qui s'opposent donc à des méthodes plus 'violentes' de la thérapie du cancer: celles qui découpent (chirurgie), brûlent (radiothérapie) ou empoisonnent (chimiothérapie) les tumeurs malignes. ${ }^{6}$

L'immunologie d'aujourd'hui - comme celle de la fin du XIX siècle - oscille entre des métaphores guerrières qui décrivent les mécanismes d'immunité comme une arme défensive, et des représentations du 'système immunitaire' comme un des mécanismes physiologiques de base de l'organisme. Si les premières images sont plus fréquentes dans les récits de vulgarisation, elles n'y sont nullement cantonnées, tandis que la vision physiologique de l'immunologie entre de plus en plus dans le domaine public. Récemment, Donna Haraway, l'historienne et théoricienne du féminisme nord-américaine, s'est appuyée sur l'image (publique) de l'immunologie comme système physiologique compliqué pour dire que cette science est l'incarnation même de l'esprit post-moderne. Les idées nouvelles de l'immunologie, telle la théorie des réseaux idiotypiques, et l'effacement des limites entre le soi et le non-soi reflètent, pour Haraway, l'instabilité et l'indétermination qui caractérisent la postmodernité. Haraway considère cependant cette vision de l'immunité comme un phénomène post-moderne, donc trés récent. C'est un argument difficile à soutenir - sauf à modifier le sens du terme post-moderne $-{ }^{7}$ puisque plusieurs pionniers de l'immunologie avaient dèjà proposé, vers 1900, que les réactions immunitaires constituent un réseau complexe et autorégulé qui inclut des réactions contre des composantes de l'organisme luimême. La post-modernité de l'immunologie, si elle existe, sera 
peut-être mieux illustrée par la difficulté de fabriquer un seul 'grand récit' soulignant ses progrès, et par la coexistence, tout au long de son histoire, de deux visions distinctes mais entremêlées des réactions immunitaires. On peut argumenter que ces deux visions correspondent aux deux visages de la médecine contemporaine: une profession héroïque qui lutte contre la maladie et la mort, et une science de pointe qui progresse grâce à une compréhension croissante des complexités du fonctionnement de l'organisme. Si, comme le conclut Anne-Marie Moulin, l'immunologie est récemment devenue "le dernier langage de la médecine", c'est sans doute - du moins en partie - grâce à la résonance entre les métaphores de l'immunologie et les identités revendiquées par la médecine contemporaine.

LÖWY, I.: 'As metáforas da imunologia: guerra e paz'. História, Ciências, Sauide - Manguinhos, III (1):7-23, mar.-jun. 1996.

A imunologia sempre recorreu a uma linguagem metafórica. Logo de início, ela oscilou entre imagens bélicas e outras que insistem na interação dos macanismos da imunidade ao conjunto das funções fisiológicas do organismo.

No final do século XIX, os glóbulos brancos eram comparados com uma 'polícia de fronteiras' encarregada de rechaçar os intrusos, com um exército formado para combater os invasores, mas também com um mecanismo fisiológico de eliminação das células envelhecidas e mortas, ocasionalmente exterminador de corpos estranhos, os anticorpos eram descritos como sendo armas muito poderosas e mortíferas, mas também como fazendo parte integrante dos mecanismos que permitem a assimilação dos alimentos pelas células.

Esta dualidade das imagens próprias da imunidade permanece ainda em nosso dias. $O$ artigo analisa a emergência e o desenvolvimento dessas imagens, relacionando-as com a redefinição da imunologia como ciência do self e do não-self, dissecando-as enfim à luz dos recentes acontecimentos, tais como a epidemia de Aids.

PALAVRAS-CHAVE: imunologia, metáforas, regulação, infecção, história das ciências.

\section{RÉFÉRENCES}

Bergmann, $M$. et Niemann, E 1937

Besredka, A. 1901

Bieganski, Wladyslaw. 1990

Billingham, R., Brent, L. et Medawar, P. B. 1953
'Newer biological aspects of protein chemistry'. Science, 86: 187-90.

'Les autohémolysines naturelles'. Annales de l'Institut Pasteur, 15: 785-807.

Zagadnienia ogolne z teoryi nauk lekarskich. Varsovie, E. Wende, 1897. Traduction anglaise (extraits) in I. Löwy. The polish school of philosophy of medicine. From Tytus Chalubinski (1820-89) to Ludwik Fleck (1896-1961). Dordrecht, Kluwer, pp. 91-100.

'Actively acquired tolerance of foreign cells'. Nature, 172: 603-6. 
Borden, Ernest C. et Sondel, Paul $\mathrm{M}$. 1990

Bordet, Jules 1920

Burnet, F. M. 1968

Burnet, F. M. 1967

Burnet, F. M. 1957

Burnet, F. M. et Fenner, $\mathrm{F}$.

1949

Burnet, F. M.; Freeman, M.; Jackson A. V. et Lush, D. 1941

Cautinho, A.; Forni, L.; Holmberg, . D.; Ivars, F. et Vazn, N. M 1984

Citron, Julius 1910

Cole, Helen 1984

Cook, Timothy E. et Colby, David C. 1992

Daëron, Marc septembre 1988

Duclaux, Émile 1898-1901

Duclaux, Émile 1896

Ehrlich, Paul 1957

Fauci, Anthony S. 1988

Fenner, F. J. 1987

Fishel, M. A.; Richman, D. D.; Laskin, O. L. et al. 1987
'Lymphokines and cytokines as cancer treatment: immunotherapy realized'. Cancer, 65: 800-14.

Traité de l'immunité dans les maladies infectieuses. Paris, Masson, p. 4.

Changing patterns: an atypical biography. Melbourne, William Heineman.

'The immunological recognition of the self', in Nobel lectures: biology or medicine, Nobel Foundation (ed.). Amsterdam/London, Elsevier Publishing Company, vol. 3, pp. 689-701.

'Modification of Jerne's theory of antibody production using the concept of clonal selection'.Australian Journal of Science, 20: 67-8.

The production of antibodies $2^{e} e ́ d$., Melbourne, Macmillan,

The production of antibodies. Melbourne,

Macmillan, pp. 1-2.

'From antigen-centered, clonal perspective of immune responses to an organism-centered network perspective of autonomous activity in a selfreferential immune system'. Immunological Reviews, 79: 151-68.

Die Methoden der Immunodiagnostik und Immunotherapie und ihre praktische Verwentung. Leipzig, Thieme.

'Aids associated disorders pose complex therapeutic challenges'. Journal of the American Medical Association, 252: 1.987-8.

'The mass-mediated epidemics: the politics of Aids on nightly newtwork news'. In E. Fee et D. M. Fox, Aids the making of a chronic disease. Berkeley, University of California Press, pp. 84-121.

'Le système immunitaire ou connais-toi toi-même'. Conférence prononcée au Colloque de Cerisy Praxis et cognition.

Traité de microbiologie. Paris, Masson et Cie, vol. 1, pp. 735-6.

Pasteur, histoire d'un esprit. Paris, p. 391.

'Experimental researches on specific therapy'. In F. Himmelwelt; M. Marquardt et M. Dale (éds.), Collected papers. London, Pegamon Press, vol. 3, pp. 118-129.

'The human immunodeficiency virus: infectivity and mechanisms of pathogenesis'. Science, 239: pp. 617-22.

'Frank Macfarlane Burnet, 3 September 1899-31 August 1985'. Biographical memoirs of the fellows of the Royal Society, 33: pp. 101-62.

"The efficacy of aziothymidine (AZT) in treatment of patients with Aids and Aids-related complex'. New England Journal of Medicine, 317: 185-91. 
Fleck, Ludwik 1988

Fleck, Ludwik 1935

Foon, Kenneth A 1989

Haraway, Donna 1989

Herzlich, Claudine et Pierret, Janine 1988

Ho, D. D.; Pomerantz, J, et Kaplan, J. C. 1987

Hoffman, Geoffry W.; Kion, Tracy A. et Grant, Michael D. 1991

Jacobi, Daniel et Scheile, Bernard 1992

Jerne, Niels K. 1974

Jerne, $\mathrm{K}$. 1955

Levy, Jay A. 1988

Lyotard, J. F. 1988

Maddox, John 1991

Medawar, Peter B. 1977

Medawar, $\mathrm{B}$. 1945

Medawar, B.

Metchnikoff, Elie 1901

Metchnikoff, Elie 1892

Metchnikoff, Elie 1884
'O swoistych cechach myslenia serologicznego'. Communication au $X V^{\mathcal{e}}$ Congrés des médecins et biologistes polonais, Lwow, 1937. Traduction anglaise in Science in Context, 2: 343.

Entstehung 1/nd Entwicklung einer wissenchaftichen Tatsasche: Einfübrung in den Lebre vom Denkstil und Denkkollectiv. Bâle, Bruno Schwabe.

'Biological response modifiers'. Cancer Research, 49: 1.621-39.

'The biopolitics of postmodern bodies: determination of self in immune system discourse'. Differences: A Journal of Feminist Cultural Studies, 1: 1-43.

'Une maladie dans l'espace public: le Sida dans six quotidiens français'. Annales: Economie, Societés, Civilisations, 43: 1.109.

'Pathogenesis of infection with HIV'. New England Journal of Medicine, 317: 305-15.

'An idiotypic network model of Aids immunopathogenesis'. Proceeding of $t$ be National Academy of Sciences, 88: 3.060-4.

'Science in magazines, and its readers'. Public Understanding of Science, 2: 3-20.

'Towards a network theory of the immune system'. Annales d'Immunologic de l'Institut Pasteur, série C, 125: 373-89.

'The natural selection theory of antibody formation'. Proceedings of the National Academy of Sciences, 41: 849-57.

'Mysteries of HIV-challenges for therapy and prevention'.

Nattre, 333: 519-22.

Le post-modernisme raconté aux enfants. Paris, Galilée, pp. 30-1.

'Aids research turned upside down'. Nature, 353: 297.

'Experiments of use and experiments of light'. In Adolf W. Yann (éd.), Beyond tomorrow: trends and prospects in medical science. New York, Rockefeller University Press, pp. 20-3.

'The experimental study of skin grafts'. British Medical Bulletin, 3: 79-81.

'The bahavior and fate of skin homografts in rabbits'. Journal of Anatomy, 78: 176-99, 1944

L'immunité dans les maladies infectieuses. Paris, Masson, p. 4.

'La lutte pour l'existence entre les diverses parties de l'organisme'. Revue Scientifique, 11: 324 .

'Untersuchung über die intercellulare Verdauung bei Wirbellosen Thieren'. Arbeiten aus dem Zoologischen Institut der Universit $t$ zu Wien, 2: 241-68. 
Merril, J. P.; Murray, J. E.; Harrinson,

J. H. et Gild, W. 1995

Moulin, Anne Marie 1991

Moulin, Anne Marie et Silverstein,

Arthur M. 1989

Owen, R. D. 1945

Pasteur, Louis 1980

Rather, L. J. 1970

Richet, Charles 1967

Root-Berenstein, Robert S.

1990

Rosenberg,

Steven A. May 1990

Rosenberg,

Steven A. 1988

Roux, E. et Chamberland, C. 1887

Silverstein, Arthur. 1989

Tauber, Alfred I. et Chernyak, Leon 1991

Volberding, $\mathrm{P}$. 1987

Zinger, John L. et Stites, Daniel P. 1986
'Successful transplantation of human kidney between identical twins'. Journal of the American Medical Association, 160: 277-82.

Le dernier langage de la médecine: Histoire de l'immunologie de Pasteur au Sida. Paris, PUF, pp. 341-76.

'History of immunophysiology'. In J. J. Oppenheim (éd.), Immunopathology. Oxford, Oxford University Press.

'Immunogenetic consequences of vascular anastomoses between cattle twins'. Science, 102: 400-3.

Comptes rendus de l'Académie des Sciences. Paris, 91, p. 315.

'Review of the reprinted editions of Metchnikoff's lectures on the comparative pathology of inflamation and immunity in infectious diseases'. Medical History, 14:409-12.

'Anaphylaxis'. In Nobel lectures: physiology or medicine. Nobel Foundation (éd.). Amsterdam/London, Elsevier Publishing Company, vol. I, pp. 469-490.

'Do we know the cause(s) of Aids?'. Perspectives in biology and medicine, 33: $480-500$

'Adaptive immunotherapy for cancer'. Scientific American, 34-41: 35.

'The development of new immunotherapies for the treatment of cancer using interleukin-2'. Annals of Surgery, 208: 121-35.

Annales de l'Institut Pasteur, 1, p. 561.

A bistory of immunology. San Diego, Academic Press, pp. 1-23, 82-3, 214-51.

Metchnikoff and the origins of immunology: from metaphor to teory. New York/Oxford, Oxford University Press, p. 206.

'Aids: variations on the theme of cellular immune deficiency'. Bulletin de l'Institut Pasteur, 85: 87-94.

'Hypohthesis: Aids is an autoimmune disease directed at the immune system and triggered by a lymphotropic virus'. Clinical Immunology and Immunopathology, 41: 305-13.

Recebido para publicação em janeiro de 1996. 